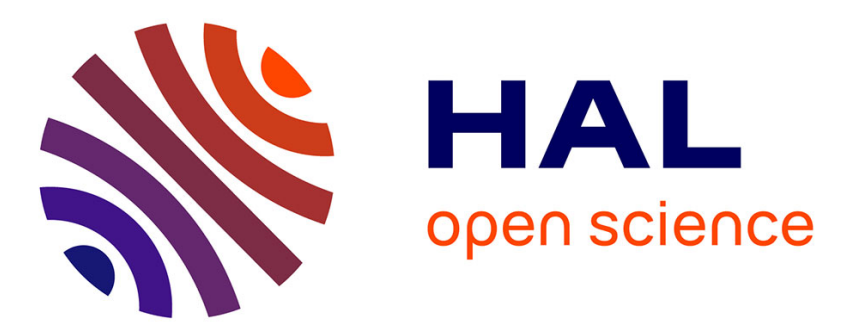

\title{
A new recursive method for subspace identification of noisy systems : EIVPM
}

Guillaume Mercère, Stéphane Lecoeuche, Christian Vasseur

\section{To cite this version:}

Guillaume Mercère, Stéphane Lecoeuche, Christian Vasseur. A new recursive method for subspace identification of noisy systems: EIVPM. 13th IFAC Symposium on System Identification, SYSID'03, Mar 2003, Rotterdam, Netherlands. pp.CDROM. hal-00091352

\section{HAL Id: hal-00091352 https://hal.science/hal-00091352}

Submitted on 6 Sep 2006

HAL is a multi-disciplinary open access archive for the deposit and dissemination of scientific research documents, whether they are published or not. The documents may come from teaching and research institutions in France or abroad, or from public or private research centers.
L'archive ouverte pluridisciplinaire HAL, est destinée au dépôt et à la diffusion de documents scientifiques de niveau recherche, publiés ou non, émanant des établissements d'enseignement et de recherche français ou étrangers, des laboratoires publics ou privés. 


\title{
A NEW RECURSIVE METHOD FOR SUBSPACE IDENTIFICATION OF NOISY SYSTEMS : EIVPM
}

\author{
Guillaume Mercère ${ }^{*, * *}$ Stéphane Lecoeuche ${ }^{*, * * *}$ \\ Christian Vasseur* \\ * Laboratoire $I^{3} D$ - Bâtiment P2, USTL - 59655 Villeneuve \\ d'Ascq - France \\ ** EIPC, Campus de la Malassise - BP39 - 62967 Longuenesse \\ Cedex-France-gmercere@eipc.fr \\ *** Ecole des Mines de Douai - Rue Charles Bourseul - BP 838 - \\ 59508 Douai Cedex-France
}

\begin{abstract}
In this article, a new recursive identification method based on subspace algorithms is proposed. This method is directly inspired by the Propagator Method used in sensor array signal processing to estimate directions of arrival (DOA) of waves impinging an antenna array. Particularly, a new quadratic criterion and a recursive formulation of the estimation of the subspace spanned by the observability matrix are presented. The problem of process and measurement noises is solved by introducing an instrumental variable within the minimized criterion.
\end{abstract}

Keywords: Identification algorithms; Recursive estimation; State-space models;

Subspace methods; Subspace tracking; Quadratic criterion

\section{INTRODUCTION}

Subspace state-space system identification (4SID) methods have been particularly developed during the last two decades (Moonen et al., 1989; Verhaegen, 1994; Van Overschee and De Moor, 1996). These techniques are well adapted to identify a state-space representation of a multivariable time-invariant system from measured I/O data. Contrary to PEM (Ljung, 1999), they don't require any nonlinear regression. They only need few structural parameters and are based on robust numerical tools such as QR factorization and Singular Value Decomposition (SVD). However, these tools, which are appropriate for off-line identification, are difficult to implement on-line due to their computational complexity. In fact, in many online identification scenarios, it is desirable to update the model as time goes on with the minimal computational cost. Recent researches on recursive algorithms for subspace identification have been developed to avoid the application of the SVD.
The most convincing on-line results (Gustafsson, 1997; Lovera, 1998; Oku and Kimura, 2002) have been obtained by introducing some techniques used in signal processing to find the directions of arrivals (DOA). One goal of this field of research is the conception of low computational cost adaptative algorithms for the location of moving sources. The method mainly used in recursive subspace identification is the Yang's algorithm PAST (Projection Approximation Subspace Tracking) (Yang, 1995). In this case, a minimization problem is considered with respect to a fourth-order cost function so as to retrieve the signal subspace from I/O data. A projection approximation is introduced in order to reduce the minimization task to an exponentially weighted least-square problem (Yang, 1995). Thus, recursive least-squares (RLS) methods can be used to find the signal subspace.

Unfortunately, these recursive methods are based on an approximation. In order to overcome this imprecision, a new quadratic cost function is proposed in this 
paper. The minimization of this second-order criterion leads to the subspace spanned by the observability matrix (i.e. the signal subspace). This new algorithm is inspired from the Propagator Method (PM) (Munier and Delisle, 1991). This technique proposes the decomposition of the observability matrix into two parts which can be easily extracted from the data. Thus, the properties of the linear propagator operator are adapted to estimate the signal subspace.

In their original form, the linear methods such as PAST or PM need strong hypotheses on the disturbances acting on the studied process. In fact, it must be assumed that the noise-covariance matrix is proportional to the identity. During any problem of identification, the reachable I/O data are most of the time disturbed at the same moment by measurement and process noises. The obtaining of reliable estimations from such data can only be realized if the effects of the noise terms are removed. The proposed solution to treat this problem is to introduce an instrumental variable in the minimized criterion in order to eliminate the effect of perturbations without losing the informations contained in the I/O data.

\section{BACKGROUND OF THE SUBSPACE RECURSIVE IDENTIFICATION TECHNIQUES}

\subsection{Problem formulation and notations}

Consider a $n^{\text {th }}$ order causal linear time-invariant statespace model with $l$ undisturbed outputs and $m$ undisturbed inputs, collected respectively in $\tilde{\mathbf{y}}(t)$ and $\tilde{\mathbf{u}}(t)$ :

$$
\left\{\begin{aligned}
\mathbf{x}(t+1) & =\mathbf{A x}(t)+\mathbf{B} \tilde{\mathbf{u}}(t)+\mathbf{w}(t) \\
\tilde{\mathbf{y}}(t) & =\mathbf{C x}(t)+\mathbf{D} \tilde{\mathbf{u}}(t)
\end{aligned}\right.
$$

where $\mathbf{w}(t) \in \mathbb{R}^{n}$ is the process noise. The measured input and output signals are modeled as:

$$
\begin{array}{r}
\mathbf{u}(t)=\tilde{\mathbf{u}}(t)+\mathbf{f}(t) \\
\mathbf{y}(t)=\tilde{\mathbf{y}}(t)+\mathbf{v}(t)
\end{array}
$$

with $\mathbf{f}(t) \in \mathbb{R}^{m}$ and $\mathbf{v}(t) \in \mathbb{R}^{l}$ the measurement noises. All these three noises are assumed to be zero-mean white noise and statistically independent of the past noise-free input $\tilde{\mathbf{u}}$. Furthermore, the measurement noises $\mathbf{f}(t)$ and $\mathbf{v}(t)$ are assumed to be independent of the state $\mathbf{x}(t)$.

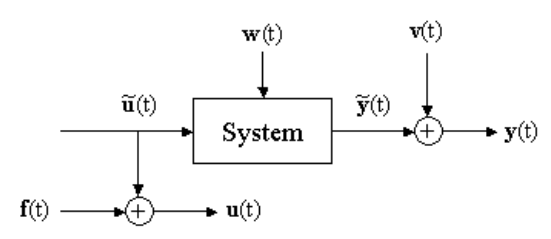

Fig. 1. Block schematic illustration of the studied system.

The problem is to estimate recursively a state-space realization from the updates of the disturbed I/O data $\mathbf{u}(t)$ and $\mathbf{y}(t)$. For that purpose, introduce the stacked $l \alpha \times 1$ output vector:

$$
\mathbf{y}_{\alpha}(t)=\left[\mathbf{y}^{T}(t) \cdots \mathbf{y}^{T}(t+\alpha-1)\right]^{T}
$$

where $\alpha>n$ is a user-defined integer. From the statespace representation (1) and (2), it is easy to show recursively that the output vector verifies (the stacked input and noise vectors are defined in the same way than $\left.\mathbf{y}_{\alpha}(t)\right)$ :

$$
\begin{aligned}
\mathbf{y}_{\alpha}(t)= & \boldsymbol{\Gamma}_{\alpha \mathbf{x}(t)+\boldsymbol{\Phi}_{\alpha} \mathbf{u}_{\alpha}(t)} \\
& \underbrace{-\boldsymbol{\Phi}_{\alpha} \mathbf{f}_{\alpha}(t)+\boldsymbol{\Psi}_{\alpha} \mathbf{w}_{\alpha}(t)+\mathbf{v}_{\alpha}(t)}_{\mathbf{b}_{\alpha}(t)}
\end{aligned}
$$

where $\Gamma_{\alpha} \in \mathbb{R}^{l \alpha \times n}$ is the observability matrix of the system:

$$
\boldsymbol{\Gamma}_{\alpha}=\left[\mathbf{C}^{T}(\mathbf{C A})^{T} \cdots\left(\mathbf{C A}^{\alpha-1}\right)^{T}\right]^{T}
$$

$\boldsymbol{\Phi}_{\alpha} \in \mathbb{R}^{l \alpha \times m}$ and $\boldsymbol{\Psi}_{\alpha} \in \mathbb{R}^{l \alpha \times n}$ are two lower triangular Toeplitz matrices. Furthermore, introduce a notation useful in the following sections :

$$
\mathbf{z}_{\alpha}(t)=\mathbf{y}_{\alpha}(t)-\boldsymbol{\Phi}_{\alpha} \mathbf{u}_{\alpha}(t)=\boldsymbol{\Gamma}_{\alpha} \mathbf{x}(t)+\mathbf{b}_{\alpha}(t)
$$

The first stage of subspace methods is typically the extraction of the observability matrix from I/O data. Indeed, from this matrix, it is possible to extract the state-space matrices of the system (Van Overschee and De Moor, 1996). In relation (4), the extended observability matrix spans a $n$-dimensional signal subspace which focuses on the informations about the direct relation between the output and state vectors. The various subspace techniques differ in the way the observability matrix is estimated. The extraction of a low dimensional subspace from a large space is, most of the time, performed by an SVD. This fundamental step is the main problem of recursive 4SID formulation. In fact, the SVD is computationally burdensome to update in an adaptative algorithm. The first researches on this topic suggested to perform the SVD on compressed I/O data matrices in order to decrease the computational load (Verhaegen and Deprettere, 1991; Cho et al., 1994). However, it was always necessary to apply the SVD at every update. An efficient method to avoid this mathematical tool in system identification was firstly proposed in (Gustafsson, 1997). Under the assumption that the order of the system is a priori known, T. Gustafsson suggested to update an estimate of the extended observability matrix thanks to the use of an algorithm named PAST.

\subsection{Review of PAST}

PAST (Projection Approximation Subspace Tracking) was originally introduced into array signal processing by Yang (Yang, 1995). In order to correctly understand the principle of this method, it is useful to present the context of the sensor array signal processing. The sensor array signal model can be written as:

$$
\mathbf{z}(t)=\Gamma(\theta) \mathbf{s}(t)+\mathbf{b}(t)
$$


where $\mathbf{z}(t) \in \mathbb{C}^{M \times 1}$ is the output of the $M$ sensors of the antenna at time $t, \Gamma(\theta) \in \mathbb{C}^{M \times n}$ the steering matrix for a direction of arrival vector $\theta, \mathbf{s}(t) \in \mathbb{C}^{n \times 1}$ the vector of the $n(n<M)$ random impinging waves and $\mathbf{b}(t) \in \mathbb{C}^{M \times 1}$ the measurement noise. B. Yang proposed an unconstrained cost function to estimate the range of $\boldsymbol{\Gamma}(\boldsymbol{\theta})\left((.)^{H}\right.$ denotes the Hermitian transposition, $\|$.$\| the Euclidean vector norm and E[$.$] the$ expectation operator):

$$
J(\mathbf{W})=E\left\|\mathbf{z}-\mathbf{W} \mathbf{W}^{H} \mathbf{z}\right\|^{2}
$$

and proved that it has neither local minimum nor maximum except for a unique global minimum which corresponds to the signal subspace. Thus, the minimisation of (8) provides an expression of $\boldsymbol{\Gamma}(\boldsymbol{\theta})$ in a particular basis. In order to update this matrix recursively, it was proposed to replace the expectation operator in (8) with an exponentially weighted sum:

$$
J(\mathbf{W}(t))=\sum_{i=1}^{t} \lambda^{t-i}\left\|\mathbf{z}(i)-\mathbf{W}(t) \mathbf{W}^{H}(t) \mathbf{z}(i)\right\|^{2}
$$

where $\lambda$ is a forgetting factor $(0<\lambda \leq 1)$ ensuring that past data are downweighted (tracking capability). The key idea of PAST is to approximate $\mathbf{W}(t)^{H} \mathbf{z}(i)$ in (9) with:

$$
\mathbf{h}(i)=\mathbf{W}^{H}(i-1) \mathbf{z}(i)
$$

This so-called projection approximation lets the criterion (9) be quadratic in $\mathbf{W}(t)$ :

$$
\bar{J}(\mathbf{W}(t))=\sum_{i=1}^{t} \lambda^{t-i}\|\mathbf{z}(i)-\mathbf{W}(t) \mathbf{h}(i)\|^{2}
$$

Assuming that the covariance of the measurement noise $\mathbf{b}(t)$ is proportional to the identity matrix, it is also possible to estimate the signal subspace by using efficient recursive tools like RLS algorithms.

T. Gustafsson (1997) was the first to use this criterion in order to estimate the subspace spanned by the observability matrix. In fact, assuming that an estimate $\hat{\boldsymbol{\Phi}}_{\alpha}(t-1)$ of the Toeplitz matrix $\boldsymbol{\Phi}_{\alpha}$ can be constructed, in the same way than (4), the following approximation can be considered:

$$
\check{\mathbf{z}}_{\alpha}(t)=\mathbf{y}_{\alpha}(t)-\hat{\boldsymbol{\Phi}}_{\alpha}(t-1) \mathbf{u}_{\alpha}(t) \simeq \boldsymbol{\Gamma}_{\alpha} \mathbf{x}(t)+\mathbf{b}_{\alpha}(t)
$$

Then, the terms of (12) can easily be connected with those of (7) (cf. tab. 1). Thus, assuming that the noisecovariance of $\mathbf{b}_{\alpha}$ is proportional to the identity matrix (e.g. $\mathbf{f}(t)=\mathbf{w}(t)=0$ and $\left.\operatorname{Cov}(\mathbf{v})=\sigma^{2} \mathbf{I}\right)$, it is possible to apply the PAST criterion to (12) so as to estimate the observability matrix.

Table 1. Relations between signal processing and subspace identification

$$
\begin{array}{ll}
\mathbf{z}_{\alpha}(t) \in \mathbb{R}^{l \alpha \times 1} & \mathbf{z}(t) \in \mathbb{C}^{M \times 1} \\
\boldsymbol{\Gamma}_{\alpha} \in \mathbb{R}^{l \alpha \times n} & \Gamma(\theta) \in \mathbb{C}^{M \times n} \\
\mathbf{x}(t) \in \mathbb{R}^{n \times 1} & \mathbf{s}(t) \in \mathbb{C}^{n \times 1} \\
\mathbf{b}_{\alpha}(t) \in \mathbb{R}^{l \alpha \times 1} & \mathbf{b}(t) \in \mathbb{C}^{M \times 1}
\end{array}
$$

With the PAST criterion, the signal subspace is derived by minimizing the modified cost function
$\bar{J}(\mathbf{W})$ instead of $J(\mathbf{W})$. Hence, the estimated columnsubspace is slightly different from the one reachable with the original cost function. Theoretically, the columns of $\mathbf{W}$ minimizing the criterion $J(\mathbf{W})$ are orthonormal. Even if this property is not necessary to extract the state-space matrices, the minimization of $\bar{J}(\mathbf{W})$ leads to a matrix having columns that are not exactly orthonormal. This property evolves during the recursive minimization since, under some conditions, the minimizer of $\bar{J}(\mathbf{W})$ converges to a matrix with orthonormal columns (Yang, 1995). This evolution can be interpreted as a slow change of basis which does not guarantee that $\Gamma_{\alpha}(t)$ and $\Gamma_{\alpha}(t-1)$ are in the same state-space basis. This corruption could pose a problem during the extraction of the estimates $\hat{\mathbf{A}}, \hat{\mathbf{B}}$, $\hat{\mathbf{C}}$ and $\hat{\mathbf{D}}$. In order to avoid these difficulties due to the use of an approximate algorithm, a new quadratic criterion is proposed in the next section.

\section{A NEW QUADRATIC SUBSPACE TRACKING CRITERION}

The new proposed cost function is inspired by a signal processing method for source bearing estimation. This technique, named the Propagator Method (PM) (Munier and Delisle, 1991), is used to find the direction of arrival without requiring the eigendecomposition of the cross-spectral matrix of the received signals (Marcos et al., 1995).

\subsection{The Propagator Method}

The propagator is a linear operator which provides the decomposition of the observation space into a noise subspace and a source subspace. In order to well understand the key point of this method, consider again the sensor array signal model (7). Assuming that the steering matrix $\Gamma$ is of full rank, $n$ rows of $\Gamma$ are linearly independent, the others being expressed as a linear combination of these $n$ rows. Under this hypothesis and after a reorganisation of the sensors outputs so that the first $n$ rows of $\Gamma$ are linearly independent, it is possible to partition the steering matrix according to:

$$
\left.\Gamma=\left[\begin{array}{l}
\Gamma_{1} \\
\Gamma_{2}
\end{array}\right]\right\} \in \begin{aligned}
& \mathbb{C}^{n \times n} \\
& \} \in \mathbb{C}^{M-n \times n}
\end{aligned}
$$

The propagator is the unique linear operator $\mathbf{P} \in$ $\mathbb{C}^{n \times M-n}$ defined as follows:

$$
\Gamma_{2}=\mathbf{P}^{H} \Gamma_{1}
$$

From the works of S. Marcos et al. (1995) on the source bearing estimation, on the basis of the similarity between (6) and (7) (cf. tab. 1) and under the hypothesis that $\{\mathbf{A}, \mathbf{C}\}$ is observable, the extended observability matrix can be decomposed in the following way:

$$
\left.\left.\boldsymbol{\Gamma}_{\alpha}=\left[\begin{array}{l}
\boldsymbol{\Gamma}_{\alpha_{1}} \\
\boldsymbol{\Gamma}_{\alpha_{2}}
\end{array}\right]\right\} \in \mathbb{R}^{n \times n}\right\} \in \mathbb{R}^{l \alpha-n \times n}
$$


where $\Gamma_{\alpha_{1}}$ is the block of the $n$ independent rows and $\Gamma_{\alpha_{2}}$ the matrix of the $l \alpha-n$ others. Thus, there is a unique $\mathbf{P} \in \mathbb{R}^{n \times l \alpha-n}$ such as:

$$
\Gamma_{\alpha_{2}}=\mathbf{P}^{T} \Gamma_{\alpha_{1}}
$$

It is also easy to verify that:

$$
\boldsymbol{\Gamma}_{\alpha}=\left[\begin{array}{l}
\boldsymbol{\Gamma}_{\alpha_{1}} \\
\boldsymbol{\Gamma}_{\alpha_{2}}
\end{array}\right]=\left[\begin{array}{c}
\mathbf{I}_{n} \\
\mathbf{P}^{T}
\end{array}\right] \boldsymbol{\Gamma}_{\alpha_{1}}=\mathbf{Q}_{s} \boldsymbol{\Gamma}_{\alpha_{1}}
$$

This means that the columns of $\Gamma_{\alpha}$ are linear combinations of the columns of $\mathbf{Q}_{s}$. Thus:

$$
\operatorname{span}\left\{\mathbf{Q}_{s}\right\}=\operatorname{span}\left\{\boldsymbol{\Gamma}_{\alpha}\right\}
$$

The new quadratic criterion proposed in this paper is based on this property (18). Indeed, this equality implies, from the knowledge of the propagator $\mathbf{P}$, the ability to find an expression of the observability matrix in a particular basis. Thus, assuming that the order $n$ is known, an estimation of the subspace spanned by the observability matrix is available by estimating $\mathbf{P}$. For that purpose, consider the equation (6). After an initial reorganisation such that the first $n$ rows of $\boldsymbol{\Gamma}_{\alpha}$ are linearly independent, the following partition can be introduced:

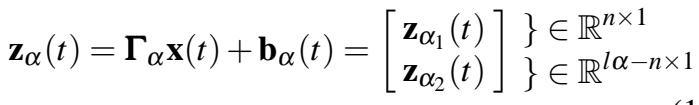

In the no-noise case, it is easy to show that:

$$
\mathbf{z}_{\alpha_{2}}=\mathbf{P}^{T} \mathbf{z}_{\alpha_{1}}
$$

In the presence of noise, this relation holds no longer. An estimation of $\mathbf{P}$ can however be obtained by minimizing the following cost function:

$$
J(\hat{\mathbf{P}})=E\left\|\mathbf{z}_{\alpha_{2}}-\hat{\mathbf{P}}^{T} \mathbf{z}_{\alpha_{1}}\right\|^{2}
$$

the unicity of $\hat{\mathbf{P}}$ being ensured thanks to the convexity of this criterion.

The criterion (21) is, by definition, quadratic and reduces the determination of the range of $\boldsymbol{\Gamma}_{\alpha}$ to the estimation of an $l \alpha-n \times n$ matrix. Its minimization is possible by using techniques such as RLS or TLS algorithms (Ljung, 1999). However, these techniques give biased estimates when the noise $\mathbf{b}_{\alpha}(t)$ is not white. In order to obtain an unbiased estimation of the signal subspace, an instrumental variable is introduced in the previous criterion so as to be applicable even if colored disturbances act on the system, as done by Gustafsson (1997).

\subsection{Instrumental variable tracking}

Since the covariance matrix of the noise $\mathbf{b}_{\alpha}$ is rarely proportional to the identity matrix, it is necessary to modify the criterion (21) so as to be applicable with colored disturbances. This correction is realized by introducing an instrumental variable $\boldsymbol{\xi}(t) \in \mathbb{R}^{\gamma \times 1}(\gamma \geq$ $n$ ) in (21), assumed not be correlated with the noise but enough correlated with $\mathbf{x}(t)$ :

$$
J_{I V}(\hat{\mathbf{P}})=E\left\|\mathbf{z}_{\alpha_{2}} \boldsymbol{\xi}^{T}-\hat{\mathbf{P}}^{T} \mathbf{z}_{\alpha_{1}} \boldsymbol{\xi}^{T}\right\|^{2}
$$

Since only a finite number of data are accessible in practice, replacing the expectation operator with a finite exponentially weighted sum, the previous criterion becomes:

$J_{I V}(\hat{\mathbf{P}}(t))=\sum_{i=1}^{t} \lambda^{t-i}\left\|\mathbf{z}_{\alpha_{2}}(i) \boldsymbol{\xi}^{T}(i)-\hat{\mathbf{P}}^{T}(t) \mathbf{z}_{\alpha_{1}}(i) \boldsymbol{\xi}^{T}(i)\right\|^{2}$

Assuming an instrumental variable can be constructed, the minimization of (23) can be realized by two different manners according to the number of instruments in $\boldsymbol{\xi}$. Indeed, if $\gamma=n$, using the matrix inversion lemma, it is possible to find a first IVPM algorithm:

$$
\begin{aligned}
\mathbf{K}(t) & =\frac{\boldsymbol{\xi}^{T}(t) \mathbf{R}(t-1)}{\lambda+\boldsymbol{\xi}^{T}(t) \mathbf{R}(t-1) \mathbf{z}_{\alpha_{1}}(t)} \\
\mathbf{P}^{T}(t) & =\mathbf{P}^{T}(t-1)+\left[\mathbf{z}_{\alpha_{2}}(t)-\mathbf{P}^{T}(t-1) \mathbf{z}_{\alpha_{1}}(t)\right] \mathbf{K}(t) \\
\mathbf{R}(t) & =\frac{1}{\lambda}\left[\mathbf{R}(t-1)-\mathbf{R}(t-1) \mathbf{z}_{\alpha_{1}}(t) \mathbf{K}(t)\right]
\end{aligned}
$$

where $\mathbf{R}(t)=\left\{E\left[\mathbf{z}_{\alpha_{1}}(t) \boldsymbol{\xi}^{T}(t)\right]\right\}^{-1}=\mathbf{C}_{\mathbf{z}_{\alpha_{1}}}^{-1} \xi(t)$.

In (Söderström and Stoica, 1989), it was proved that the accuracy of the estimate obtained from an instrumental variable method increases with the number of instruments. It would be interesting to improve the efficiency of the previous algorithm by increasing the number of used instruments (i.e. $\gamma>n$ ). In that case, the minimization of the criterion (23) appeals to a technique named the Extended Instrumental Method (Friedlander, 1984). The application of such a technique gives the recursive updating formulae of appendix A, named EIVPM, the main step being:

$$
\mathbf{P}^{T}(t)=\mathbf{P}^{T}(t-1)+\left(\mathbf{g}(t)-\mathbf{P}^{T}(t-1) \Psi(t)\right) \mathbf{K}(t)
$$

The complexity of this new algorithm is comparable to the one of PAST or its by-products.

Remark 1. From the second iteration, the updating of the estimated subspace has always the same writing:

$$
\hat{\boldsymbol{\Gamma}}_{\alpha}=\left[\begin{array}{c}
\mathbf{I}_{n} \\
\hat{\mathbf{P}}^{T}
\end{array}\right]
$$

This means that, after a short transient period, the recursive estimation is made in the same state-space basis. This property is an important asset for the extraction of the state-space matrices.

\section{RECURSIVE IDENTIFICATION SCENARIO AND INSTRUMENTAL VARIABLE CHOICE}

In this section are presented the main stages of the recursive update of the state-space matrices. First of all, assume that an estimate of the system matrices is available at time $t-1$. It is then possible to build an estimate of the matrix $\boldsymbol{\Phi}_{\alpha}(t-1)$ by noticing, for example, that the first block column of $\hat{\boldsymbol{\Phi}}_{\alpha}(t-1)$ expresses itself simply according to $\hat{\mathbf{B}}(t-1), \hat{\mathbf{D}}(t-1)$ and $\hat{\boldsymbol{\Gamma}}_{\alpha}(t-1)$, the other blocks being simple partitions 
of this one. From this estimate, the approximation $\check{\mathbf{z}}_{\alpha}(t)$ (cf. eq. (12)) can be constructed.

The following step is the setting up of the instrumental variable. Since the available data are temporal data, the easiest way to get a good instrumental variable is to use delayed I/O data. As far as figure 1 is concerned, the instruments must be correlated with present and future data $\tilde{\mathbf{y}}_{\alpha}$ and/or $\tilde{\mathbf{u}}_{\alpha}$ but uncorrelated with $\mathbf{w}_{\alpha}, \mathbf{f}_{\alpha}$ and $\mathbf{v}_{\alpha}$. Introduce the following notation:

$$
\mathbf{u}_{\beta}(t)=\left[\begin{array}{lll}
\mathbf{u}^{T}(t-\beta-\mu) & \cdots & \mathbf{u}^{T}(t-1-\mu)
\end{array}\right]
$$

where $\beta$ and $\mu$ are user-defined integers. This variable, as $\mathbf{y}_{\beta}$, represents the available past $\mathrm{I} / \mathrm{O}$ data from which the instrument vector can be constructed. These data are not noise-free. It is then necessary to study the correlation between the past and future reachable signals to focus on the informative data uncorrelated with the disturbances. By considering table 2, the

Table 2. Correlation between the available I/O data.

\begin{tabular}{cccccc} 
& $\tilde{\mathbf{u}}_{\beta}$ & $\mathbf{f}_{\beta}$ & $\mathbf{w}_{\beta}$ & $\tilde{\mathbf{y}}_{\beta}$ & $\mathbf{v}_{\beta}$ \\
\hline$\tilde{\mathbf{u}}_{\alpha}$ & 0 & 0 & 0 & 0 & 0 \\
$\mathbf{f}_{\alpha}$ & 0 & 0 & 0 & 0 & 0 \\
$\mathbf{w}_{\alpha}$ & 0 & 0 & 0 & 0 & 0 \\
$\tilde{\mathbf{y}}_{\alpha}$ & $\checkmark$ & 0 & $\checkmark$ & $\checkmark$ & 0 \\
$\mathbf{v}_{\alpha}$ & 0 & 0 & 0 & 0 & 0
\end{tabular}

only past data uncorrelated with present and future disturbances but correlated with the informative signal $\tilde{\mathbf{y}}_{\alpha}$ is the past measured input vector $\mathbf{u}_{\beta} \equiv\left\{\tilde{\mathbf{u}}_{\beta}, \mathbf{f}_{\beta}\right\}$. Thus, the choice of the instrumental variable is:

$$
\boldsymbol{\xi}(t)=\mathbf{u}_{\beta}(t)
$$

where $\beta$ is chosen so that $\gamma=m \beta>n$ and $\mu$ so that the temporal correlation length of the instrumental variable is larger than that of the noise.

By feeding the EIVPM algorithm (cf. Appendix A) with $\check{\mathbf{z}}_{\alpha}(t)$ and $\boldsymbol{\xi}(t)$, a new estimation of the observability matrix is reached. Then, the last stage consists in the extraction of system-matrices. Firstly, the estimation of $\hat{\mathbf{A}}(t)$ and $\hat{\mathbf{C}}(t)$ is made as in the nonrecursive case thanks to the shift-invariance structure of $\boldsymbol{\Gamma}_{\alpha}$. Given the estimates $\hat{\mathbf{A}}(t)$ and $\hat{\mathbf{C}}(t)$, the matrices $\hat{\mathbf{B}}(t)$ and $\hat{\mathbf{D}}(t)$ can be obtained from a linear regression problem ((Van Overschee and De Moor, 1996) chap. $4)$, the second part of the regressor being calculable recursively (e.g. (Lovera, 1998)).

\section{EXAMPLES}

In this section, the results of a numerical simulation are presented to illustrate the performances of the new recursive algorithm EIVPM compared with the extended instrumental version of the PAST method named EIVPAST (Gustafsson, 1997).

Consider the following fourth-order MIMO system found in the appendix diskette of (Van Overschee and De Moor, 1996) (sta_demo.m):

$$
\begin{aligned}
& \mathbf{x}_{k+1}=\left[\begin{array}{cccc}
0.603 & 0.603 & 0 & 0 \\
-0.603 & 0.603 & 0 & 0 \\
0 & 0 & -0.603 & -0.603 \\
0 & 0 & 0.603 & -0.603
\end{array}\right] \mathbf{x}_{k} \\
& +\left[\begin{array}{cc}
1.1650 & -0.6965 \\
0.6268 & 1.6961 \\
0.0751 & 0.0591 \\
0.3516 & 1.7971
\end{array}\right] \tilde{\mathbf{u}}_{k}+\left[\begin{array}{cc}
0.1242 & -0.0895 \\
-0.0828 & -0.0128 \\
0.0390 & -0.0968 \\
-0.0225 & 0.1459
\end{array}\right] \mathbf{e}_{k}
\end{aligned}
$$

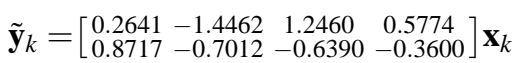

$$
\begin{aligned}
& +\left[\begin{array}{cc}
-0.1356 & -1.2704 \\
-1.3493 & 0.9846
\end{array}\right] \tilde{\mathbf{u}}_{k} \\
& \operatorname{Cov}(\mathbf{e})=\left[\begin{array}{cc}
0.0176 & -0.0267 \\
-0.0267 & 0.0497
\end{array}\right]
\end{aligned}
$$

To be in the case of figure 1 , measurement noises are added to the undisturbed I/O data. Thus, the input $\tilde{\mathbf{u}}$, a Gaussian white noise sequence with variance 1 , is contaminated with a zero-mean white noise sequence $\mathbf{f}$ with variance 0.64 . The output $\tilde{\mathbf{y}}$ is also disturbed by a Gaussian white measurement noise such as the signalto-noise ratio equals $16 d B$. Now, let the initial system matrices be randomly generated under the constraint that the absolute value of the maximum eigenvalue of $\hat{\mathbf{A}}(0)$ is less than 1 (stability requirement). To reduce the effect of this random initialization, consider the following forgetting factor:

$$
\lambda(t)=\min \left\{\lambda_{0} \lambda(t-1)+1-\lambda_{0}, \lambda_{\text {final }}\right\}
$$

For this example, $\lambda_{0}=\lambda(t=0)=0.99$ and $\lambda_{\text {final }}=$ 0.999 . Furthermore, the following user-defined quantities are applied: $\alpha=\beta=6$ and $\mu=1$.

In figure 2 , the eigenvalues trajectories obtained respectively with the new EIVPM algorithm and EIVPAST are presented. In both cases, the eigenvalue estimations are close to the true values.

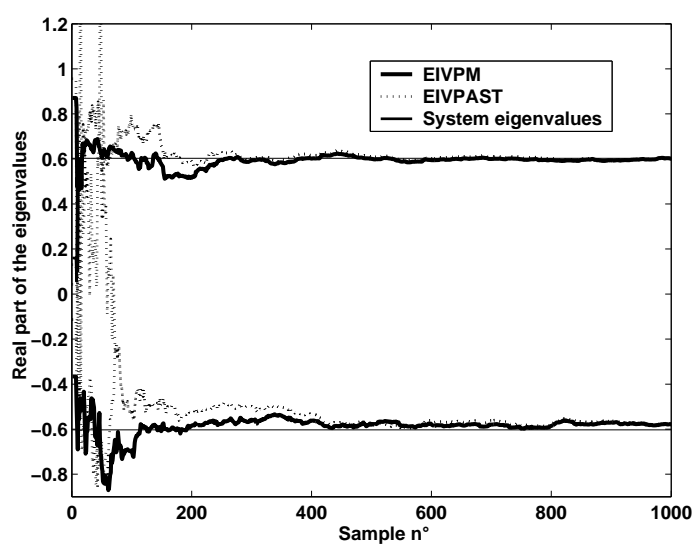

Fig. 2. Estimated real part of the eigenvalues using the new EIVPM algorithm and EIVPAST.

Even if both results seems to be equally accurate (both methods present the same behaviour after 500 loops), the speed of convergence of EIVPM is higher than the one of EIVPAST. This asset is confirmed in figure 3 since, for each eigenvalue, the normalized mean quadratic error for EIVPM is globally smaller than the one supplied by EIVPAST.

The figure 4 emphasizes the real benefit of EIVPM in term of accuracy, the initialization step being aside. By avoiding to take account of the 100 first iterations, the normalized mean quadratic error for both methods 

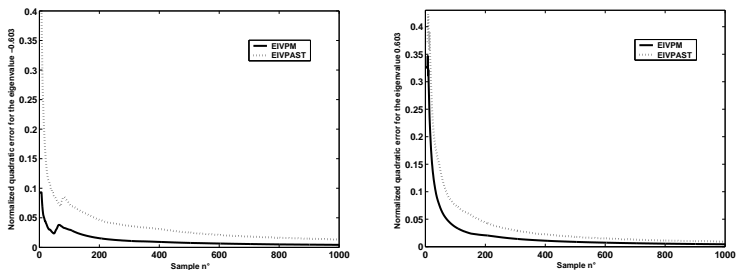

Fig. 3. Normalized mean quadratic error of both eigenvalues.

is plotted again. The mean quadratic error of EIVPM is constantly smaller than with EIVPAST (it can be selectively up to eight times smaller). Thus, EIVPM is more accurate both during and after the initialization stage.
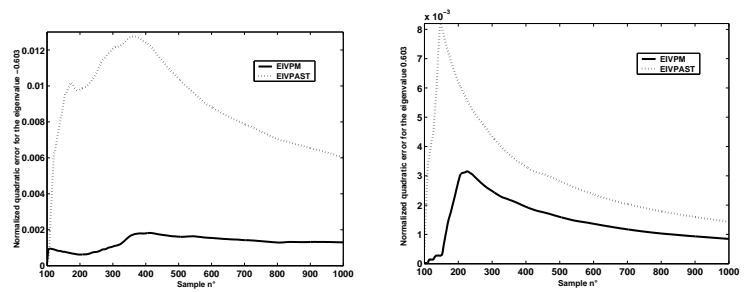

Fig. 4. Normalized mean quadratic error of both eigenvalues aside from the initialization step.

\section{CONCLUSION}

In this paper, the problem of recursive identification of a state-space system with process and measurement noises is considered. In order to update the model online with the minimal computational cost, a new recursive algorithm is presented. Under the assumption that the state order is a priori known, a new quadratic criterion has been proposed to estimate recursively the subspace spanned by the observability matrix. The use of an instrumental technique has allowed to annihilate the effects of the disturbances during the recursive subspace identification. The performances of the EIVPM algorithm have been compared with those of EIVPAST on a simulation example. The good results of EIVPM in terms of accuracy and speed of convergence have been underlined.

\section{REFERENCES}

Cho, Y. M., G. Xu and T. Kailath (1994). Fast recursive identification of state space models via exploitation displacement structure. Automatica 30(1), 45-60.

Friedlander, B. (1984). The overdetermined recursive instrumental variable method. IEEE Transactions on Automatic Control 4, 353-356.

Gustafsson, T. (1997). Recursive system identification using instrumental variable subspace tracking. In: the $11^{\text {th }}$ IFAC Symposium on System Identification. Fukuoka, Japan.
Ljung, L. (1999). System identification. Theory for the user. $2^{\text {nd }}$ ed.. PTR Prentice Hall Information and System Sciences Series. T. Kailath, Series Editor.

Lovera, M. (1998). Subspace identification methods: theory and applications. $\mathrm{PhD}$ thesis. Politecnico di Milano.

Marcos, S., A. Marsal and M. Benidir (1995). The propagator method for source bearing estimation. Signal Processing 42(2), 121-138.

Moonen, M., B. De Moor, L. Vandenberghe and J. Vandewalle (1989). On and off line identification of linear state space models. International Journal of Control 49(1), 219-232.

Munier, J. and G. Y. Delisle (1991). Spatial analysis using new properties of the cross spectral matrix. IEEE Transactions on Signal Processing 39(3), 746-749.

Oku, H. and H. Kimura (2002). Recursive 4SID algorithms using gradient type subspace tracking. Automatica 38(6), 1035-1043.

Söderström, T. and P. Stoica (1989). System identification. Prentice Hall International Series in Systems and Control Engineering. New York.

Van Overschee, P. and B. De Moor (1996). Subspace identification for linear systems. Theory, implementation, applications. Kluwer Academic Publishers.

Verhaegen, M. (1994). Identification of the deterministic part of mimo state space models given in innovations form from input output data. Automatica 30, 61-74.

Verhaegen, M. and E. Deprettere (1991). A fast, recursive mimo state space model identification algorithm. In: the $30^{\text {th }}$ Conference on Decision and Control. pp. 1349-1354.

Yang, B. (1995). Projection approximation subspace tracking. IEEE Transactions on Signal Processing 43(1), 95-107.

\section{Appendix A. THE EIVPM ALGORITHM}

$$
\begin{aligned}
& \mathbf{P}^{T}(t)=\mathbf{P}^{T}(t-1)+\left(\mathbf{g}(t)-\mathbf{P}^{T}(t-1) \mathbf{\Psi}(t)\right) \mathbf{K}(t) \\
& \mathbf{g}(t)=\left[\hat{\mathbf{C}}_{\mathbf{z}_{\alpha_{2}} \boldsymbol{\xi}}(t-1) \boldsymbol{\xi}(t) \mathbf{z}_{\alpha_{2}}(t)\right] \\
& \boldsymbol{\Lambda}(t)=\left[\begin{array}{cc}
-\boldsymbol{\xi}^{T}(t) \boldsymbol{\xi}(t) & \lambda \\
\lambda & 0
\end{array}\right] \\
& \mathbf{q}(t)=\hat{\mathbf{C}}_{\mathbf{z}_{\alpha_{1}} \boldsymbol{\xi}}(t-1) \boldsymbol{\xi}(t) \quad \boldsymbol{\Psi}(t)=\left[\mathbf{q}(\mathbf{t}) \mathbf{z}_{\alpha_{1}}(t)\right] \\
& \mathbf{K}(t)=\left(\boldsymbol{\Lambda}(t)+\boldsymbol{\Psi}^{T}(t) \mathbf{M}(t-1) \boldsymbol{\Psi}(t)\right)^{-1} \boldsymbol{\Psi}^{T}(t) \mathbf{M}(t-1) \\
& \hat{\mathbf{C}}_{\mathbf{z}_{\alpha_{1}} \boldsymbol{\xi}}(t)=\lambda \hat{\mathbf{C}}_{\mathbf{z}_{\alpha_{1}} \boldsymbol{\xi}}(t-1)+\mathbf{z}_{\alpha_{1}}(t) \boldsymbol{\xi}^{T}(t) \\
& \hat{\mathbf{C}}_{\mathbf{z}_{\alpha_{2}} \boldsymbol{\xi}}(t)=\lambda \hat{\mathbf{C}}_{\mathbf{z}_{\alpha_{2}} \boldsymbol{\xi}}(t-1)+\mathbf{z}_{\alpha_{2}}(t) \boldsymbol{\xi}^{T}(t) \\
& \mathbf{M}(t)=\frac{1}{\lambda^{2}}(\mathbf{M}(t-1)-\mathbf{M}(t-1) \mathbf{\Psi}(t) \mathbf{K}(t)) \\
& \text { with } \mathbf{M}(t)=\left(\hat{\mathbf{C}}_{\mathbf{z}_{\alpha_{1}}} \boldsymbol{\xi}(t) \hat{\mathbf{C}}_{\mathbf{z}_{\alpha_{1}} \boldsymbol{\xi}}^{T}(t)\right)^{-1}
\end{aligned}
$$

